\title{
Observações sobre a modulação da camada limite atmosférica marinha devida à passagem de um sistema frontal no Atlântico Sudoeste
}

\author{
Observations on the marine atmospheric boundary layer modulation due to the passage of a \\ frontal system in the Southwestern Atlantic \\ Fernando Rossato ${ }^{1}$ e Ronald Buss de Souza ${ }^{2}$ \\ ${ }^{1}$ Universidade Federal de Santa Maria - UFSM \\ rossato.fernando@hotmail.com \\ ${ }^{2}$ Centro Regional Sul de Pesquisas Espaciais, Instituto Nacional de Pesquisas Espaciais - CRS/INPE \\ ronald@dsr.inpe.br
}

\begin{abstract}
Resumo
Entre os dias 6 a 9 de dezembro de 2012, o Navio Oceanográfico Alpha-Crucis realizou estações oceanográficas no Oceano Atlântico Sudoeste em apoio aos projetos SAMOC e ACEx. Durante o cruzeiro de pesquisa, o navio realizou medições com o lançamento de balões meteorológicos. A partir de dados coletados pelas radiossondas, foi possível verificar a variabilidade da camada limite atmosférica marinha (CLAM) na área e período de estudo. A partir de dados meteorológicos e oceanográficos, foi possível estimar os fluxos de calor latente e sensivel a partir de parametrizações bulk. Durante um episódio de formação de um ciclone extratropical, os resultados desse trabalho demonstram o impacto das condições sinóticas sobre a evolução da CLAM. Na condição pré-frontal, verificou-se uma estratificação da CLAM. Os fluxos de calor latente e sensível dirigidos do oceano para a atmosfera foram baixos ou negativos (da atmosfera para o oceano). Na condição pós-frontal, a CLAM apresentou-se bem desenvolvida, com uma altura de $1200 \mathrm{~m}$ e os fluxos de calor latente e sensivel do oceano para a atmosfera foram mais intensos. Essas observações são fundamentais para ampliar o entendimento dos processos físicos que ocorrem na interface oceano-atmosfera e tem importância primária para a previsão do tempo e clima na região sul-sudeste do Brasil.
\end{abstract}

Palavras-chave: Camada Limite Atmosférica Marinha. Fluxos de calor.

\begin{abstract}
Between 6-9 December 2012, the Oceanographic Vessel Alpha Crucis took oceanographic stations in the Southwestern Atlantic Ocean in support to both SAMOC and ACEx projects. During the research cruise, the vessel performed measurements with the release of weather balloons. From the data collected by the radiosondes, it was possible to verify the variability of the marine atmospheric boundary layer $(M A B L)$ in the area and period of study. From meteorological and oceanographic data, it was possible to estimate the latent and sensible heat fluxes from bulk parameterization. During an episode of formation of an extratropical cyclone, the results, of this work demonstrate the impact of the synoptic conditions influencing the evolution of the MABL. During the prefrontal condition a stratification of the MABL was verified. The latent and sensible heat fluxes directed from the ocean to the atmosphere were low or negative (from the atmosphere to the ocean). During the post frontal condition, the MABL was well developed with a $1200 \mathrm{~m}$ height and the latent and sensible heat fluxes from the ocean to the atmosphere were more intense. These observations are fundamental to widen our understanding on the physical processes occurring at the ocean-atmosphere interface and have primary importance for the weather and climate forecast of the south-southwestern region of Brazil.
\end{abstract}

Keywords: Marine Atmospheric Boundary Layer. Heat Fluxes. 


\section{Introdução}

Os oceanos são um importante componente do sistema climático global, que com sua grande capacidade térmica, armazenam a energia solar para que esta possa ser transferida, na forma de calor sensível e latente, para a atmosfera. (PEIXOTO et al., 1992).

Os processos de interação do sistema acoplado oceano-atmosfera ocorrem através das interações na interface da camada limite oceânica e camada limite atmosférica marinha (CLAM), onde se processam as trocas de momentum e energia (PEZZI et al., 2005, 2009; ACEVEDO et al., 2010; CAMARGO et al., 2013). A temperatura da superfície do mar (TSM) exerce um papel importantíssimo nesse complexo mecanismo de interações, pois é por meio da diferença de temperatura entre a superfície do mar e do ar que a energia em forma de fluxos de calor é trocada entre a atmosfera e o oceano (WELLS, 1997).

A interação entre massas de água e a atmosfera pode ser descrita através da representação dos fluxos de calor latente e sensível entre o oceano e a atmosfera. O Oceano Atlântico Sudoeste e sua região costeira são considerados regiões chave para melhor entender e prever o tempo e o clima da região sul e sudeste do Brasil (PEZZI e SOUZA, 2009).

O Oceano Atlântico Sudoeste também é apontado como uma das regiões de maior ocorrência de ciclogênese na América do Sul (GAN e RAO 1991), é comum nesta região a passagem de distúrbios transientes, como ciclones extratropicais e frentes frias. Segundo Acevedo et al. (2010) a CLAM é também modulada pelos padrões de advecção atmosférica.

O objetivo deste trabalho é descrever o comportamento da estrutura vertical da CLAM na interface oceano-atmosfera e os fluxos de calor entre o oceano e a atmosfera sobre o Oceano Atlântico Sudoeste a partir de dados coletados in situ pelos projetos SAMOC e ACEx durante o período entre 6 e 9 de dezembro de 2012.

\section{Material e Métodos}

Parte dos objetivos do projeto SAMOC incluem observações acerca dos parâmetros de interação oceano-atmosfera na região do Oceano Atlântico Sudoeste. Observações de campo foram tomadas a bordo do Navio Oceanográfico (NOc.) Alpha-Crucis, tendo sido realizado entre os dias 1 e 16 de dezembro de 2012. Nesse cruzeiro cobriu-se uma seção zonal ao longo de $34.5^{\circ} \mathrm{S}$, no Oceano Atlântico Sudoeste. Dados oceanográficos e meteorológicos foram obtidos ao longo da rota de navegação do navio.

A região de estudo é o Oceano Atlântico Sudoeste entre as coordenadas de $20-40^{\circ} \mathrm{S}$ e 40 -60 W. Essa região foi recoberta pela trajetória do NOc. Alpha-Crucis e dividida em quatro transectos (perfis) independentes conforme apresentado na Figura 1. Esses perfis foram selecionados a partir da derrota do navio e agrupam dados em locais do Oceano sujeitos a diferentes regimes oceanográficos e a distintas condições atmosféricas sinóticas atuantes no momento de realização do cruzeiro.

Desses quatro transecto, o presente estudo analisa aquele tomado ao longo de 34,5 (P2) durante o período entre 6 e 9 de dezembro de 2012. Esse transecto foi escolhido por apresentar pequenos contrastes de TSM, além de estar sujeito, durante o período analisado, a condição meteorológica com a formação de um ciclone extratropical.

Em colaboração com o Projeto ACEx (Atlantic Ocean Carbon Experiment), o NOc. Alpha-Crucis realizou 19 medições (P2 - 7 medições), com o lançamento de balões meteorológicos equipados com radiossondas, a fim de analisar o perfil vertical da atmosfera.

Para estabelecer a altura da CLAM utilizou-se o método adotado por Pezzi et al. (2009), que leva em consideração a mudança abrupta da temperatura potencial virtual e umidade específica nos perfis de radiossondas.

Dados meteorológicos foram coletados pela estação meteorológica automática (EMA) e dados de TSM foram coletados pelo termosalinógrafo instalados a bordo do NOc. Alpha-Crucis, durante todo o período do 


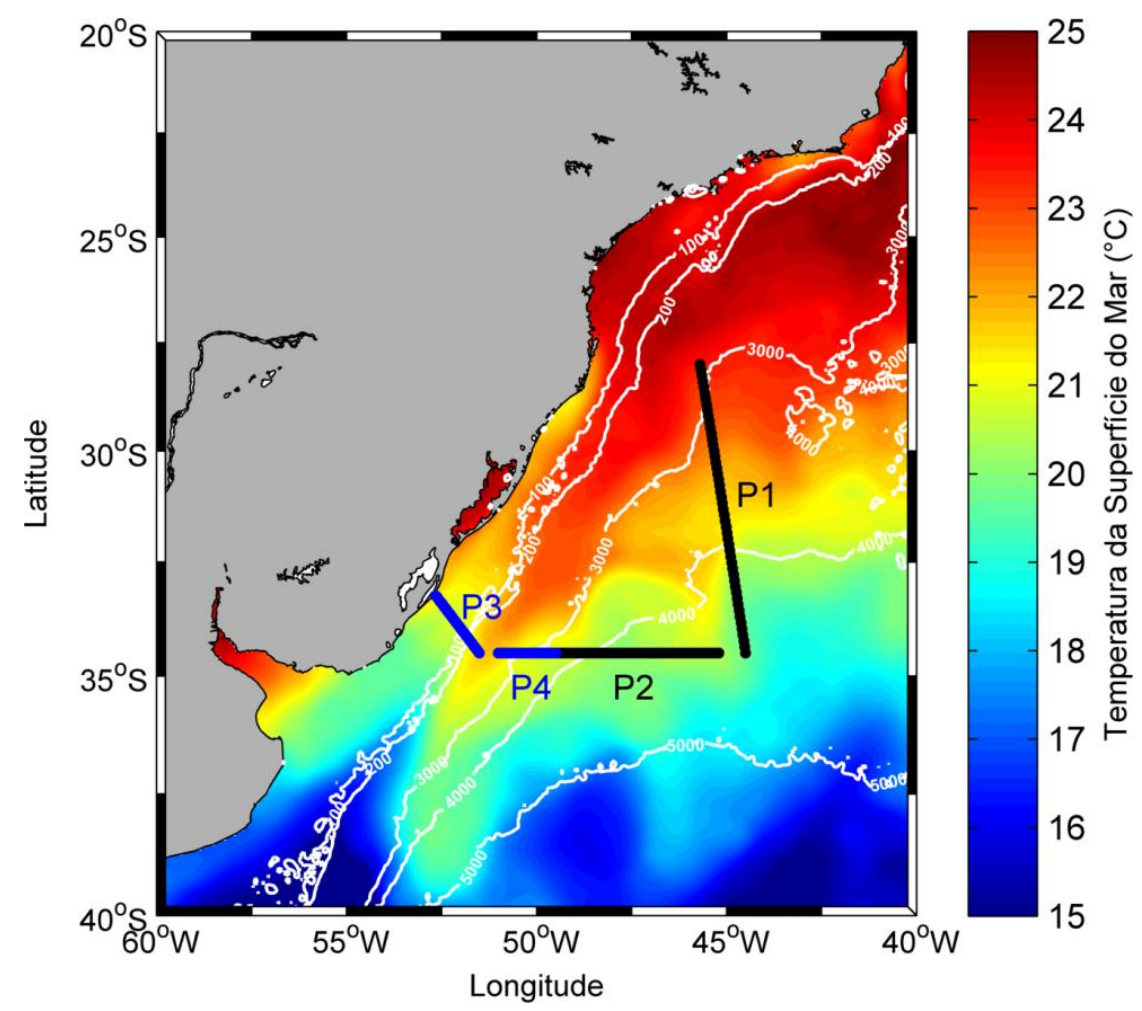

Figura 1: Imagem de TSM do GHRSST (Group for High Resolution Sea Surface Temperature) média dos dias 1-16 de dezembro de 2012 com a posição dos transectos realizadas no cruzeiro SAMOC. Em branco estão as linhas batimétricas entre $100 \mathrm{~m}$ e $4000 \mathrm{~m}$ de profundidade.

cruzeiro. Os fluxos de calor latente e sensível foram estimados ao longo do transecto seguindo o esquema proposto por Fairall et al. (1996). Para este esquema são calculadas estimativas de parametrizações dos coeficientes de transferência turbulenta de calor e equações de fluxo baseadas na teoria de similaridade de Monin-Obuklov (LIU et al., 1979 ), conhecidas como bulk formulas. Esse esquema utiliza dados de umidade do ar, velocidade do vento, temperatura do ar e TSM. Apesar do esquema de parametrização ter sido elaborado para as estimativas de fluxos de calor no Pacífico Equatorial, ele vem sendo utilizado em trabalhos recentes no Oceano Atlântico Sudoeste (PEZZI et al., 2005, 2009; ACEVEDO et al., 2010).

Para identificar os sistemas atmosféricos que atuaram no período do embarque, utilizaram-se dados de reanálise de alta resolução (SAHA et al., 2010, 2014) disponível pelo National Centers for Environmental Prediction (NCEP) Climate Forecast System (CFS) versão 2, na resolução espacial de $0.5^{\circ}$ lat/long e resolução temporal de seis horas, de pressão ao nível médio do mar (PNMM) e vento em 1000 hPa (não mostrado).

\section{Resultado e discussões}

O Transecto teve duração do dia 6 (dia Juliano 341) a 9 de dezembro de 2012 (dia Juliano 344). Foi realizado no sentido leste para oeste como apresentado na Figura 1.

A condição sinótica durante a realização do transecto 2 iniciou-se no dia 6 sob a atuação da Alta Subtropical do Atlântico Sul (ASAS). No inicio do dia 7 o experimento foi realizado sob condição pré-frontal, no setor quente de um ciclone extratropical e se aproximou com ventos intensos de norte. Ao final do dia a frente já passou pelo transecto. Esse se encontrava sob o setor frio do ciclone com escoamento predominantemente de sudoeste. No dia 8 , com predomínio da direção do vento de sudoeste, indicando uma advecção negativa de temperatura do ar na área do experimento no início do dia. Para o restante do dia, os ventos 
foram predominantemente de oeste devido à aproximação de uma circulação anticiclônica. No dia 9, o escoamento de sudoeste foi sendo afetado pela passagem de um sistema frontal frio ao sul da região sob estudo, devido à aproximação de um novo sistema de alta pressão.

Através da análise dos perfis verticais de temperatura potencial virtual, umidade específica, umidade relativa e magnitude do vento para as sete radiossondagens do $\mathrm{P} 2$, notase que os dados do transecto mostram uma atmosfera úmida e ventos em superfície com magnitudes altas quando a CLAM estava mais turbulenta, consequência da atuação do ciclone extratropical. Para as três primeiras radiossondagens, apesar da atuação da ASAS, o campo em superfície estava bem perturbado modificando a CLAM, o que resultou em uma camada mais estratificada, como mostra a radiossondagem 3 (Figura 2). $\mathrm{O}$ mesmo ocorre para a radiossondagem 4 cujos dados corroboram uma condição de transição para o pós-frontal.

Já para as três últimas radiossondagem tomadas na região pós-frontal, a CLAM apresentou uma camada de mistura espessa e turbulenta, com uma altura de $1200 \mathrm{~m}$ para a radiossonda 5 (Figura 3 ).
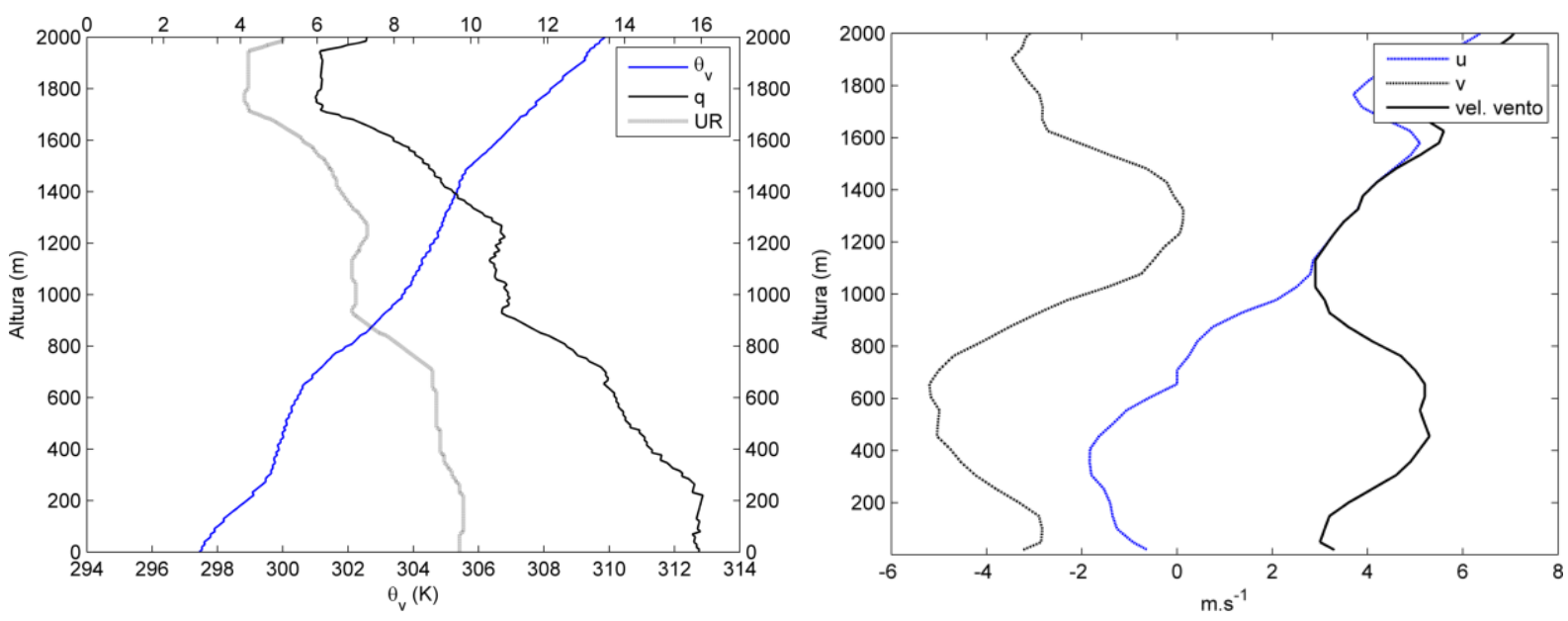

Figura 2: Perfil vertical de temperatura potencial virtual $(\mathrm{K})$, umidade específica $(\mathrm{g} / \mathrm{kg})$ e umidade relativa (x10\%) (esquerda) e magnitude do vento $(\mathrm{m} / \mathrm{s})$ (direita) para a radiossonda 3.
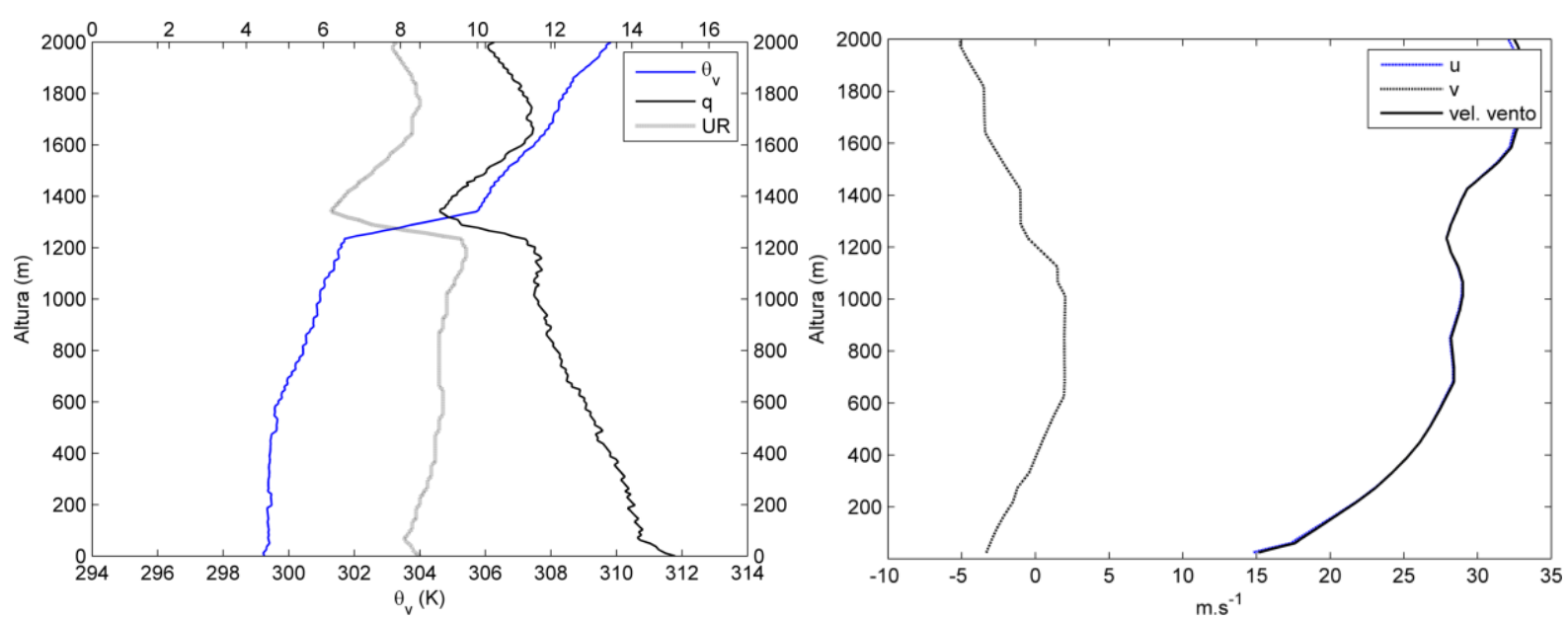

Figura 3: Perfil vertical de temperatura potencial virtual $(\mathrm{K})$, umidade específica $(\mathrm{g} / \mathrm{kg})$ e umidade relativa (x10\%) (esquerda) e magnitude do vento $(\mathrm{m} / \mathrm{s})$ (direita) para a radiossonda 5. 
Na Figura 4, é mostrada a distribuição temporal dos fluxos de calor latente e sensível no transecto, o comportamento da TSM e Tar durante a realização do $\mathrm{P} 2$, onde mostra uma queda da Tar entre os dias Julianos 342,5 a 343 dia devido a passagem do sistema frontal. Durante a realização deste transecto houve alternância entre instantes em que a TSM esteve maior do que a Tar e instantes em que a TSM esteve menor do que a Tar. Sendo assim, também ocorreu alternância no parâmetro de estabilidade do ar (Figura 4) em instantes com valores positivos, (indicando instabilidade) e instantes com valores negativos (indicando uma atmosfera estável) ao longo do transecto.

As trocas na interface oceano-atmosfera estão proximamente correlacionadas com o parâmetro de estabilidade do ar, principalmente o fluxo de calor sensível. Os resultados encontrados (Figura 4) demonstram que após a passagem do sistema, ambiente pósfrontal, os fluxos de calor latente (média de 290
$\mathrm{W} / \mathrm{m}^{2}$ e com pico de $468 \mathrm{~W} / \mathrm{m}^{2}$ ) e calor sensível (média de $18 \mathrm{~W} / \mathrm{m}^{2}$ e com pico de $45 \mathrm{~W} / \mathrm{m}^{2}$ ) indicam um intenso fluxo de calor do oceano para a atmosfera e uma CLAM instável. Por outro lado, na região pré-frontal valores mais baixos de fluxos de calor latente (média de 18 $\mathrm{W} / \mathrm{m}^{2}$ e com menor valor de $-15,8 \mathrm{~W} / \mathrm{m}^{2}$ ) e calor sensível (média de $-6 \mathrm{~W} / \mathrm{m}^{2}$ e com menor valor de $\left.-63,4 \mathrm{~W} / \mathrm{m}^{2}\right)$ e com caracterizam uma CLAM mais estável.

Esses resultados concordam com os estudos de Acevedo et al. (2010) para a região da CBM e Farias (2014) para a região costeira do sul do Brasil. Esses autores demonstraram o gradiente termal e a advecção de massas de ar associada a passagem de distúrbios transientes são mecanismos essenciais no processo da modulação da CLAM e que a passagem de uma massa de ar pós-frontal intensifica a transferência de calor do oceano para a atmosfera na busca do equilíbrio térmico e impulsionam a convecção.
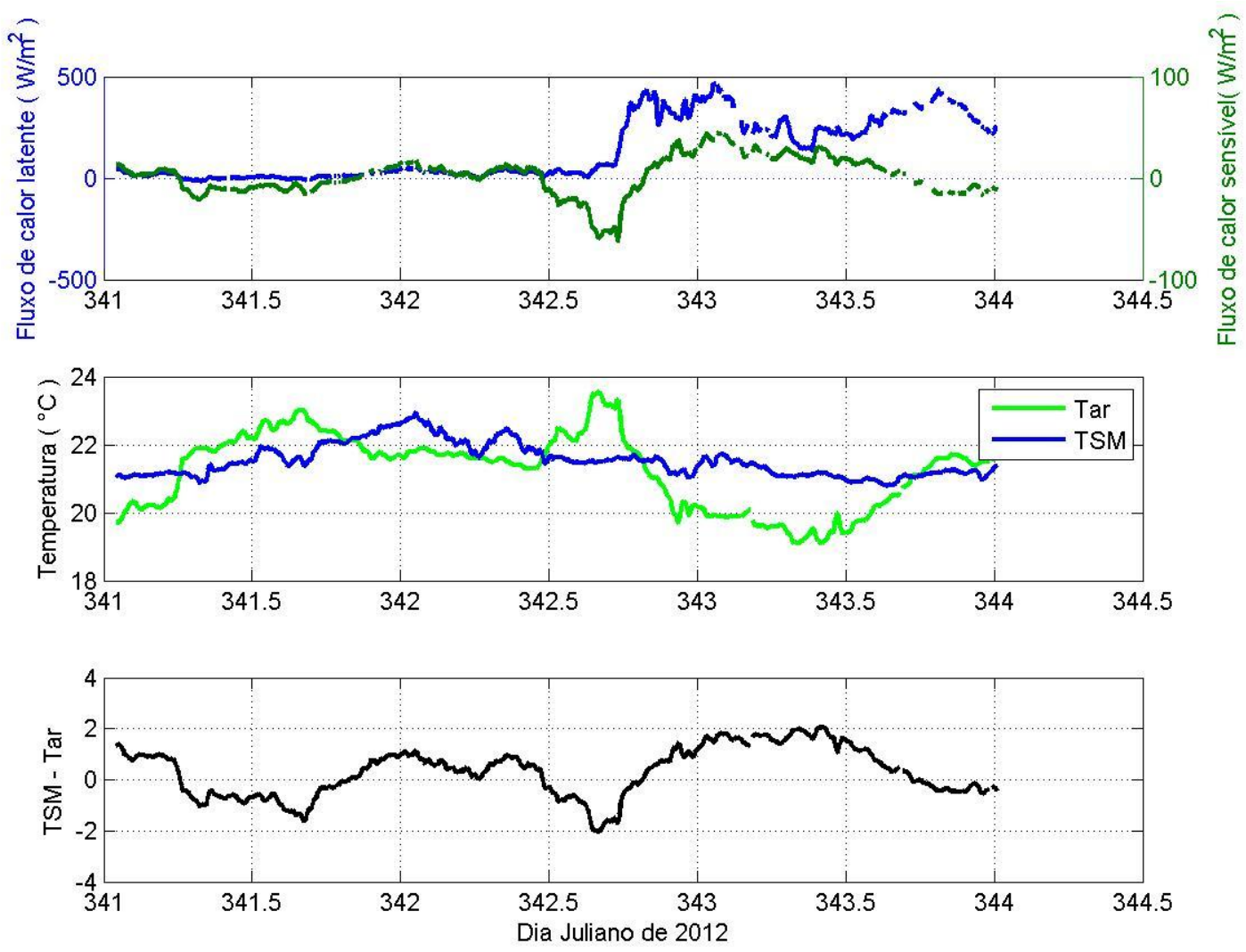

Figura 4: Calor latente $\left(\mathrm{W} / \mathrm{m}^{2}\right)$ (azul) e calor sensível $\left(\mathrm{W} / \mathrm{m}^{2}\right)$ (verde) médio de 15 minutos (acima), temperatura da superfície do mar (azul) e temperatura do ar (verde) (centro) e parâmetro de estabilidade dado pela TSM - T ar (abaixo) durante a realização do transecto, estimados com dados coletados pelos projetos SAMOC e ACEx durante o cruzeiro de pesquisa a bordo do NOc. Alpha-Crucis entre o dias 6 (dia Juliano 341 ) a 9 de dezembro de 2012 (dia Juliano 344). 


\section{Conclusões}

Neste trabalho apresentaram se resultados sobre o sistema acoplado oceano-atmosfera através de dados observacionais. Com a formação de um ciclone extratropical na região do Rio da Prata, os resultados demonstram a importância das condições sinóticas de grande escala da atmosfera influenciando a evolução da CLAM e também para a transferência de calor entre o oceano e a atmosfera.

Durante a condição pré-frontal onde verificou-se uma estratificação da CLAM. Na região pós-frontal, a CLAM apresentou-se espessa e turbulenta, com uma altura de $1200 \mathrm{~m}$. No ambiente pós-frontal, os fluxos de calor latente $\left(468 \mathrm{~W} / \mathrm{m}^{2}\right)$ e calor sensível $\left(45 \mathrm{~W} / \mathrm{m}^{2}\right)$ indicam um fluxo de calor do oceano para a atmosfera muito mais intenso em sua componente latente. Por outro lado, na região pré-frontal, valores mais baixos de fluxos de calor latente $\left(-15,8 \mathrm{~W} / \mathrm{m}^{2}\right)$ e calor sensível $(-63,4$ $\mathrm{W} / \mathrm{m}^{2}$ ) foram encontrados.

O esquema de parametrização dos fluxos de calor vem sendo amplamente utilizado em trabalhos em todo o oceano global, apesar de Fairall et al. (1996) ter sido originalmente elaborado para a região do Pacífico Equatorial. Isso pode induzir erros em algumas situações. Segundo Acevedo et al. (2010), o esquema de parametrização proposto por Fairall et al. (1996) pode ser falho em caso de extrema estabilidade da CLAM na região da CBM.

\section{Agradecimentos}

Os autores agradecem ao comandante e a tripulação do NOc. Alpha Crucis pela ajuda na coleta de dados em campo. A FAPESP pelo apoio ao projeto SAMOC (Processo No. 2011/50552-4) e ao $\mathrm{CNPq}$ pelo apoio aos projetos ACEx (Processo No. 558108/2009-1) e INCT da Criosfera (Processo No. 704222/2009). O primeiro autor agradece à CAPES pela consessão de bolsa de estudos.

\section{Referências}

Acevedo, O. C.; Pezzi, L. P.; Souza, R. B.; Anabor, V.; Degrazia, G. A. Atmospheric boundary layer adjustment to the synoptic cycle at the BrazilMalvinas Confluence, South Atlantic Ocean. Journal of Geophysical Research, v. 115, n. D22, p. 1-12, nov 2010.

Camargo, R.; Todesco, E.; Pezzi, L. P.; Souza, R. B. Modulation mechanisms of marine atmospheric boundary layer at the BrazilMalvinas Confluence region. Journal of Geophysical Research: Atmospheres, v. 118, n. 12, p. 6266-6280, 2013.

Fairall, C. W.; Bradley, E. F.; Rogers, D. P.; Edson, J. B.; Young, G. S. Bulk parameterization of air-sea fluxes for Tropical Ocean- Global Atmosphere Coupled-Ocean Atmosphere Response Experiment difference relative analysis. Journal of Geophysical Research, v. 101, p. 3747-3764, 1996.

Farias, P. C. Fluxos de calor e dióxido de carbono entre o oceano e a atmosfera na região costeira e oceânica ao sul do Brasil. 2014. 91 p. Dissertação (Mestrado) - Universidade Federal de Santa Maria, Santa Maria, RS, Brasil, 2014.

Peixoto, J. P.; Oort, A. H. Physics of Climate. New York: Springer-Verlag New York, 1992. 495p.

Pezzi, L. P.; Souza, R. B. DE; Acevado, O.; et al. Multiyear measurements of the oceanic and atmospheric boundary layers at the BrazilMalvinas confluence region. Journal of Geophysical Research, v. 114, n. D19, p. 1-19, out 2009.

Pezzi, L. P.; Souza, R. B. Variabilidade de mesoescala e interação oceano-atmosfera no Atlântico Sudoeste. In: CAVALCANTI et al. Tempo e Clima no Brasil. São Paulo: Oficina de Textos, 2009. p. 385-405. 